\title{
Near-infrared-responsive, superparamagnetic Au@Co nanochains
}

\author{
Varadee Vittur ${ }^{1, \S}$, Arati G. Kolhatkar ${ }^{1}$, Shreya Shah ${ }^{1}$, Irene Rusakova ${ }^{2}$, Dmitri Litvinov ${ }^{* 1,3}$ \\ and T. Randall Lee*1
}

\section{Full Research Paper}

\section{Address:}

${ }^{1}$ Department of Chemistry and the Texas Center for Superconductivity, University of Houston, 4800 Calhoun Road, Houston, TX 77204, USA, ${ }^{2}$ Department of Physics and the Texas Center for Superconductivity, University of Houston, 4800 Calhoun Road, Houston, TX 77204, USA and ${ }^{3}$ Department of Electrical and Computer Engineering, University of Houston, 4800 Calhoun Road, Houston, TX 77204, USA

\section{Email:}

Dmitri Litvinov* - litvinov@uh.edu; T. Randall Lee ${ }^{*}$ - trlee@uh.edu

* Corresponding author

$\S$ Deceased, February 2016

Keywords:

Au@Co; magneto-optical; nanochains; near-IR-active;

superparamagnetic
Beilstein J. Nanotechnol. 2017, 8, 1680-1687. doi:10.3762/bjnano.8.168

Received: 31 March 2017

Accepted: 18 July 2017

Published: 14 August 2017

Associate Editor: J. J. Schneider

(C) 2017 Vittur et al.; licensee Beilstein-Institut. License and terms: see end of document.

\begin{abstract}
This manuscript describes a new type of nanomaterial, namely superparamagnetic Au@Co nanochains with optical extinctions in the near infrared (NIR). The Au@Co nanochains were synthesized via a one-pot galvanic replacement route involving a redoxtransmetalation process in aqueous medium, where Au salt was reduced to form Au shells on Co seed templates, affording hollow $\mathrm{Au}$ @Co nanochains. The Au shells serve not only as a protective coating for the Co nanochain cores, but also to give rise to the optical properties of these unique nanostructures. Importantly, these bifunctional, magneto-optical Au@Co nanochains combine the advantages of nanophotonics (extinction at ca. $900 \mathrm{~nm}$ ) and nanomagnetism (superparamagnetism) and provide a potentially useful new nanoarchitecture for biomedical or catalytic applications that can benefit from both activation by light and manipulation using an external magnetic field.
\end{abstract}

\section{Introduction}

The unique properties of magnetic nanoparticles have led to diverse applications in the fields of magnetic data storage, catalysis, magnetic fluids, biosensors, drug delivery, and magnetic imaging [1-6]. Considerable efforts have been taken to tailor the magnetic properties to suit specific applications $[7,8]$. In parallel, there have been significant advances in the development of plasmonic nanoparticles (e.g., gold and silver), and efforts to tune the extinction wavelength (e.g., into the near infrared (NIR)) are ongoing [9]. Plasmonic nanoparticles exhibit intense colors and have been used in various applications, such 
as energy conversion in solar cells [10,11], biosensing [12], photothermal therapy [13], and biomedical imaging [14]. Surface modification with an inorganic coating, such as silica, can lend biocompatibility to the nanoparticles [15-18]. A gold shell on magnetic nanoparticles not only renders the nanoparticles biocompatible but also gives rise to distinct optical properties $[18,19]$. Noble metal nanoparticles, such as gold and silver, possess the unique property of surface plasmon resonance (SPR); the latter exhibit a strong extinction band in the visible region $[19,20]$. Importantly, studies have focused on the synthesis of magnetic core-plasmonic shell structures to obtain versatile, hybrid nanoparticles with dual functionality [21-23].

The NIR region of light from approximately 800 to $1200 \mathrm{~nm}$ can penetrate human tissue (i.e., the "water window") and is minimally absorbed by tissue chromophores and water [24]. Therefore, tunable plasmonic nanoparticles that can respond to NIR light and can be manipulated with a magnetic field hold great promise. Among various magnetic nanoparticles, cobalt nanoparticles have attracted much interest due to their strong magnetic properties and their greater stability toward oxidation compared to Ni- and Fe-based magnetic nanoparticles [23].

Notably, there have been a handful of studies on magnetooptical nanostructures consisting of cobalt coated with gold. Bao and co-workers synthesized magnetic $\mathrm{Co}-\mathrm{Au}$ core-shell nanoparticles [25] by reducing an organo-gold compound onto a cobalt seed with a weak reducing agent in toluene. These particles showed superparamagnetic behavior and a strong optical extinction at ca. $680 \mathrm{~nm}$. Similarly, Wetz and co-workers prepared hybrid Co-Au nanorods via decomposition of an organometallic compound under hydrogen [26]. The particles showed extinction maxima at ca. $550 \mathrm{~nm}$ and ca. $720 \mathrm{~nm}$. Liang et al. used Co nanoparticles as sacrificial templates to prepare hollow gold nanospheres [27]. The formation of these particles involved a redox-transmetalation process between the core and shell arising from the difference between their reduction potentials. Cobalt cores were oxidized by $\mathrm{H}^{+}$from aqueous $\mathrm{HAuCl}_{4}$ solution until Co nanoparticles were completely consumed, which led to the formation of the hollow gold nanostructures. The surface plasmon resonance of these particles appeared at $628 \mathrm{~nm}$.

In addition to these studies on gold-coated cobalt nanoparticles, there are, to our knowledge, only three reports of gold-coated cobalt (Au@Co) nanochains. Huang and co-workers synthesized bimetallic PtCo and AuCo magnetic nanochains, and showed how the magnetic and electronic properties could be modulated depending on the noble metal selected [28]. Duan et al. synthesized Pt-coated Co nanochains in an aqueous medium by magnetic-field induced assembly and galvanic replacement reaction, and $\mathrm{Lu}$ et al. synthesized $\mathrm{Au}$-coated Co nanochains in a similar fashion $[29,30]$. However, none of these studies explored the optical properties of the Au-coated magnetic Co nanochains. Consequently, there have been no reports characterizing both the optical and the magnetic properties of this unique class of nanomaterial.

In the present investigation, we describe a one-pot synthesis of magneticAu@Co nanochains using a wet-chemical method based on a galvanic replacement reaction. Scheme 1 shows that cobalt nanoparticles serve as seed templates over which Au salt is chemically reduced into a shell. By adding Au stock solution to suspensions of the cobalt core solution, and with the assistance of polyvinylpyrrolidone (PVP), Au@Co nanochains with a hollow interior are formed. Using this new methodology, we were able to shift the optical extinction of the generated nanoparticles to the near-infrared (NIR) region (about $900 \mathrm{~nm}$ ), which is significantly higher than the maximum of $700 \mathrm{~nm}$ observed previously for related nanostructures [28-30]. This considerable increase can be attributed to the formation of both the hollow interior and the chain structure in the present materials.

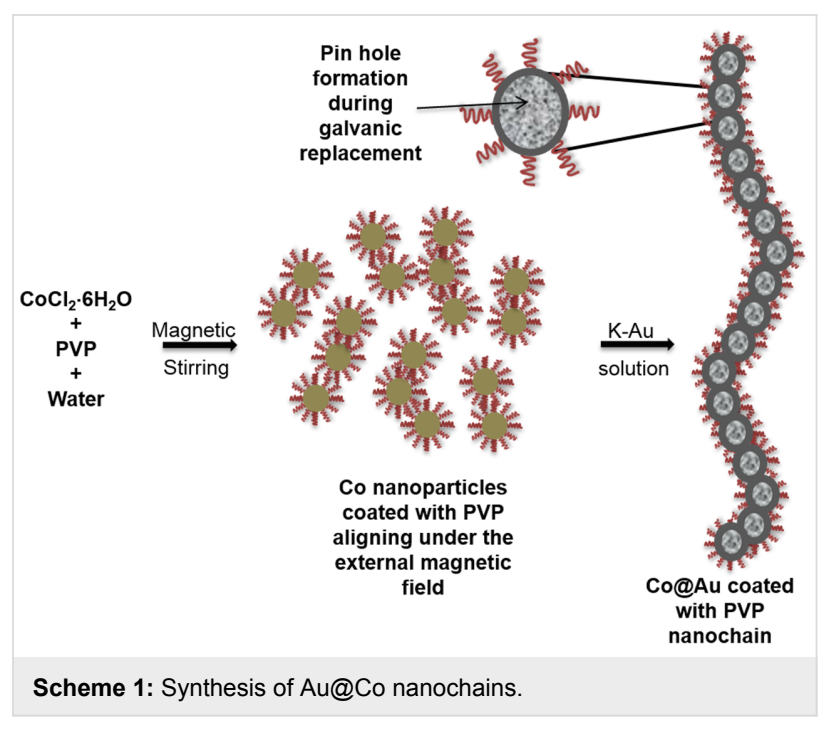

Previous research on $\mathrm{Au}$ and $\mathrm{Ag}$ nanochains demonstrated that the plasmon resonance of two interacting particles undergoes a red shift from that of a single particle because of near-field coupling. This shift in wavelength is proportional to the number of nanoparticles in the chain $[31,32]$. These new types of magnetic nanostructures find use in several applications, such as combined MRI imaging and photothermal treatment and bioseparation, that require magnetic properties and strong optical extinctions in the visible and/or near-infrared [33-35]. Our $\mathrm{Au} @$ Co nanochains are superparamagnetic at room temperature (blocking temperature of $150 \mathrm{~K}$ ) and thus offer the capacity for control and delivery under the directed influence of an 
external magnetic field. Furthermore, the NIR-responsive capability (optical extinction maximum at ca. $900 \mathrm{~nm}$ ) combined with their magnetic properties can plausibly be harnessed in nanotechnology-based biomedical applications, such as photothermally modulated drug delivery, photonic devices, and photo-thermal cancer therapy [10-13].

\section{Results and Discussion Morphology and composition of the Au@Co nanochains}

The synthesis of the Au@Co nanochains proceeded using wetchemical methods via a galvanic replacement reaction [27] Cobalt nanoparticles were used as templates for the nanochain synthesis. Because the standard reduction potentials of the $\mathrm{AuCl}_{4}{ }^{-} / \mathrm{Au}$ pair $(E=0.990 \mathrm{~V}$ vs the standard hydrogen electrode, SHE) is higher than that of the $\mathrm{Co}^{2+} / \mathrm{Co}$ pair ( $E=-0.277 \mathrm{~V}$ vs SHE), $\mathrm{AuCl}_{4}^{-}$will be reduced to $\mathrm{Au}(0)$ upon addition of the basic solution of potassium and gold $(\mathrm{K}$-gold solution) to the Co nanoparticle stock solution, leading to the formation of $\mathrm{Au} @ \mathrm{Co}$. The structure and morphology of the $\mathrm{Au} @$ Co nanochains were characterized by using transmission electron microscopy (TEM) and scanning electron microscopy (SEM) as shown in Figure 1 and Figure 2, where sub-micrometer-sized chains are readily apparent.

Figure 2 illustrates the difference in contrast between the inner and outer regions of the chains, consistent with the growth of a thin gold shell around the Co nanoparticles. The high-magnification TEM images also show that the chains are composed of distinct spherical nanoparticles, with an average outer diameter of $75 \pm 3 \mathrm{~nm}$, in close contact with each other; the shell is rough, with a typical thickness of about $14 \pm 6 \mathrm{~nm}$. Moreover, some pinholes were observed in the wall of the bimetallic nanochains, indicating the formation of a hollow nanostructure as shown in Figure 2c.

Figure 3 provides the X-ray diffraction (XRD) data of the synthesized nanochains. The XRD pattern shows peaks at
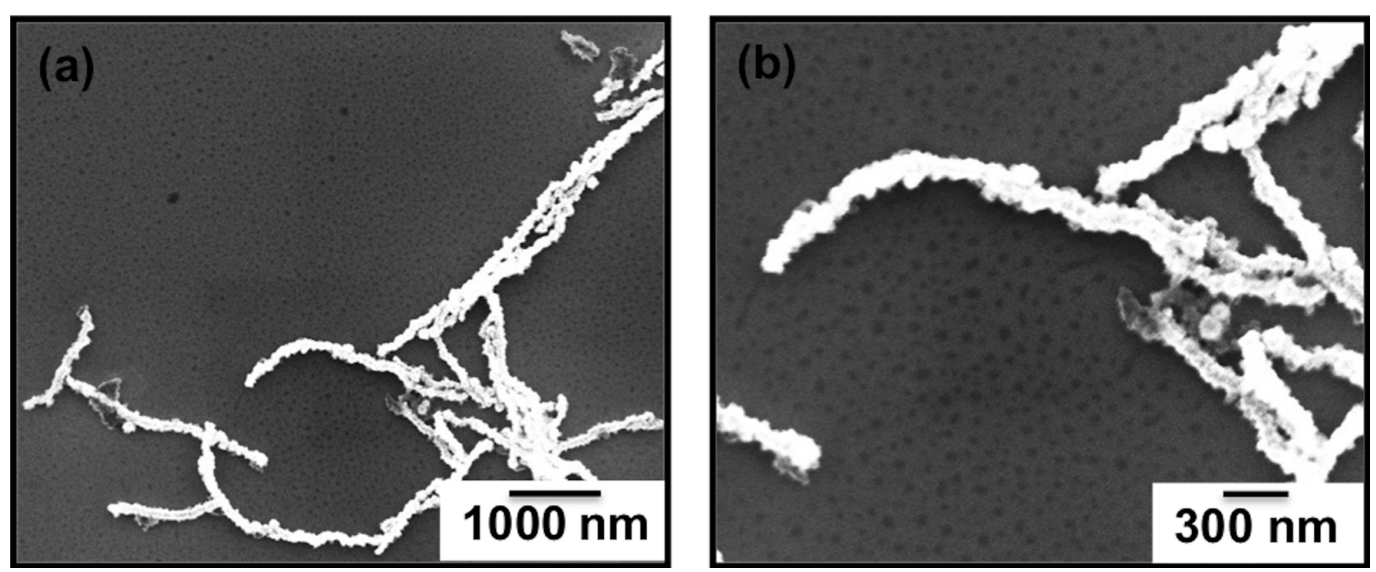

Figure 1: SEM images of the Au@Co nanochains at (a) low magnification and (b) high magnification.
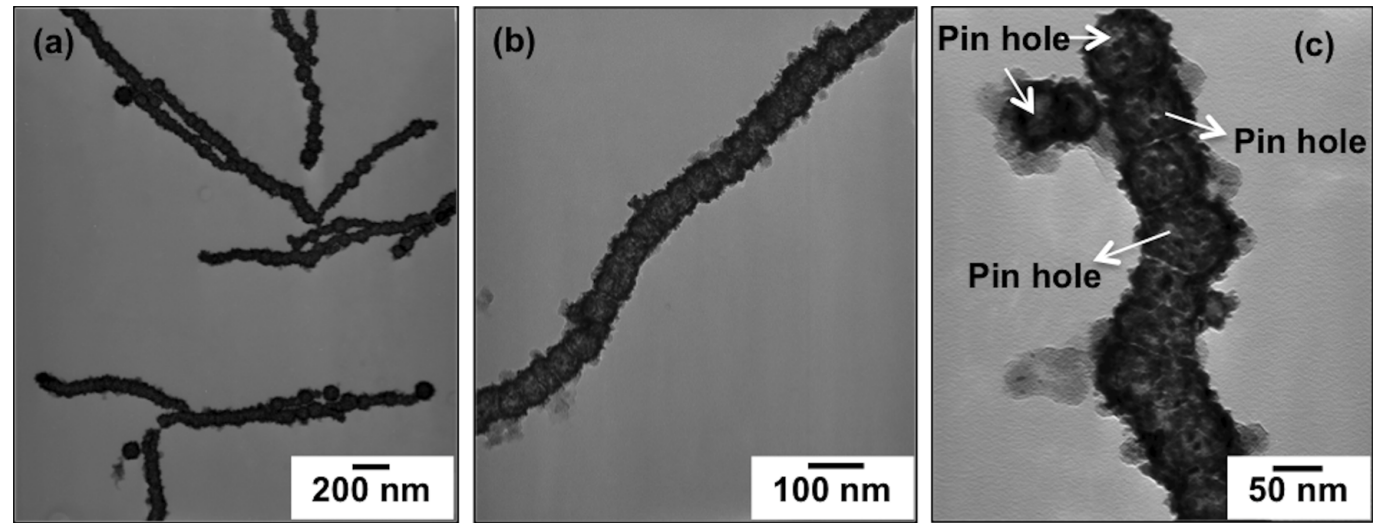

Figure 2: TEM images of the Au@Co nanochains: $(a, b)$ low-magnification and $(c)$ high magnification. 


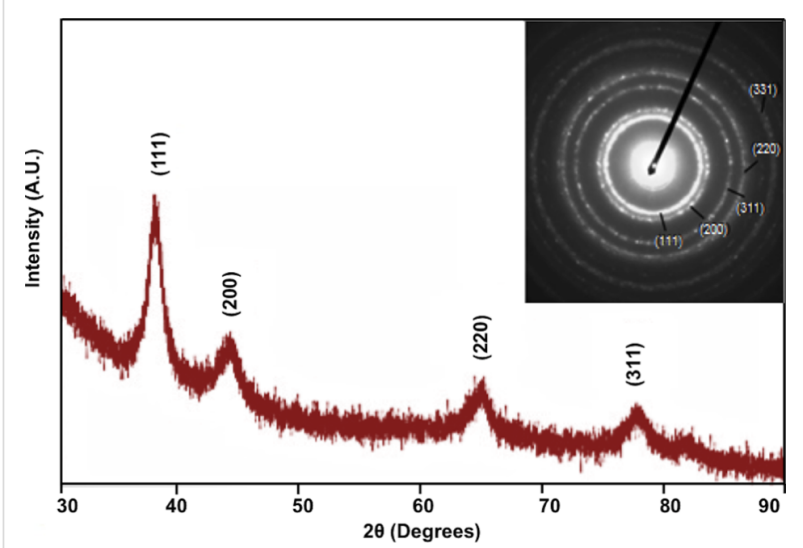

Figure 3: X-ray diffraction pattern and SAED pattern (inset) for the Au@Co nanochains.

$2 \theta=38.34^{\circ}, 46.36^{\circ}, 65.24^{\circ}$ and $77.11^{\circ}$, which correspond, respectively, to the (111), (200), (220), and (311) crystallographic planes of the face-centered cubic (fcc) structure of gold. The selected area electron diffraction (SAED) shown in the inset is also consistent with a single-phase fcc structure [36]

The chemical composition of the bimetallic nanochains was evaluated by energy dispersive X-ray spectroscopy (EDX) and $\mathrm{X}$-ray photoelectron spectroscopy (XPS). Figure 4 shows the EDX spectrum with peaks characteristic of gold $(\mathrm{M} \alpha, \mathrm{L} \alpha, \mathrm{L} \beta$, and $\mathrm{L} \gamma$ ) at 2.12, 9.71, 11.60, and $13.45 \mathrm{keV}$, respectively, and peaks characteristic of $\mathrm{Co}(\mathrm{L} \alpha$ and $\mathrm{K} \alpha)$ at 0.78 and $6.93 \mathrm{keV}$, respectively. Peaks from the supporting grid of copper are observed at 8.04 and $8.90 \mathrm{keV}(\mathrm{K} \alpha$ and $\mathrm{K} \beta)$, respectively. The peak intensity is related to the abundance of each element, and the EDX data averaged over multiple runs confirm that the approximate stoichiometry of the nanochains is $\mathrm{Co}_{27} \mathrm{Au}_{72}$.

We also used XPS to analyze the chemical composition of the nanochains (Figure 5). The XPS spectrum shows two peaks at

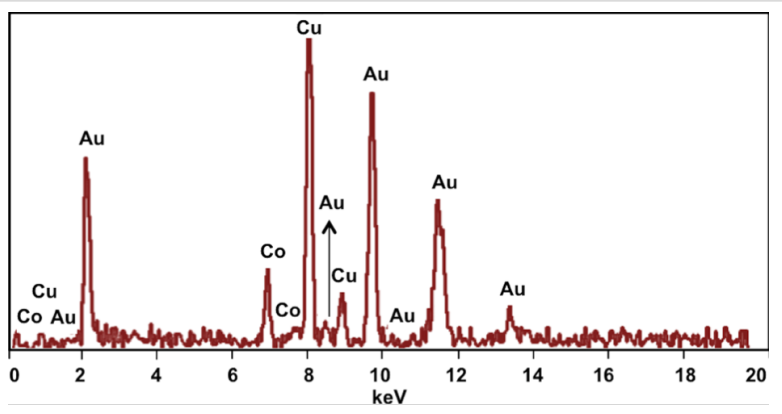

Figure 4: EDX spectrum of the Au@Co nanochains.

84.0 and $87.7 \mathrm{eV}$, which can be attributed to $\mathrm{Au} 4 \mathrm{f}$ electrons, as well as peaks at 780.2 and $795.3 \mathrm{eV}$, which can be attributed to Co $2 p$ electrons. The minor broadened peaks that were observed at binding energies of ca. $790 \mathrm{eV}$ and just above $800 \mathrm{eV}$ can be attributed to cobalt oxide [37]. Since XPS is a surface technique, the penetration depth is less than $10 \mathrm{~nm}$ [38]; therefore, we would not expect to see Co in these analyses. However, XPS studies of our gold-coated cobalt nanochains showed peaks for Co $2 \mathrm{p}_{1 / 2}$ electrons $(793 \mathrm{eV})$ and Co $2 \mathrm{p}_{3 / 2}$ electrons $(778 \mathrm{eV})$. We believe that the observation of Co by XPS was due to the existence of pinholes and/or the hollow structure of the particles, which is consistent with the TEM images.

Reports of the formation of hollow nanostructures via galvanic replacement are known [39-46]. There are also some examples where Co nanoparticles are used as templates [24,27,47,48]. By varying the surfactant added, we were able to obtain Au-coated Co nanochains. As the standard reduction potentials for the $\mathrm{AuCl}_{4}{ }^{-} / \mathrm{Au}$ pair is much higher than that of the $\mathrm{Co}^{2+} / \mathrm{Co}$ pair, Co nanoparticles were immediately oxidized to $\mathrm{Co}^{2+}$ when the $\mathrm{K}$-gold solution was added. Given the rapid nature of this replacement reaction, it is likely that nucleation of the reduced $\mathrm{Au}$ atoms led to small Au particles that eventually grew into a thin $\mathrm{Au}$ shell covering the cobalt nanoparticles. At the same
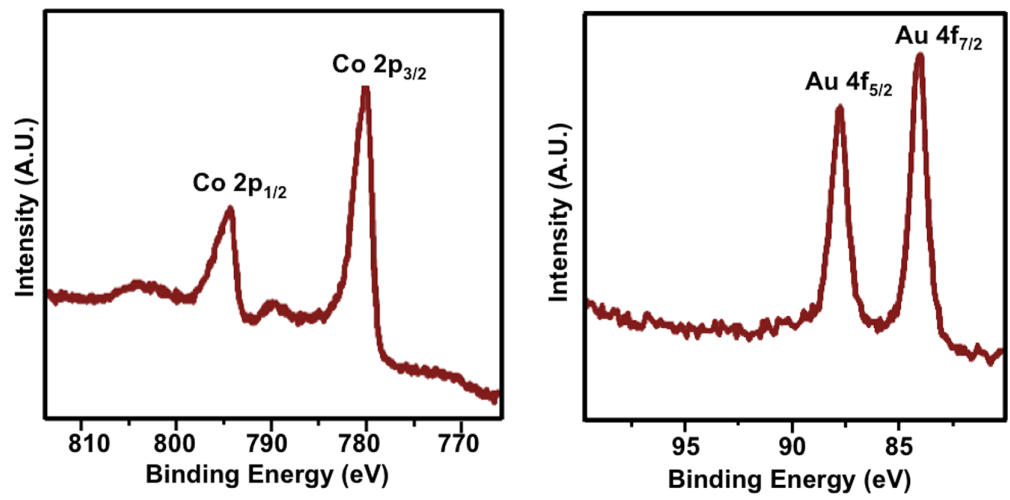

Figure 5: XPS spectra of the Au@Co nanochains. 
time, because of its extended polymeric chain structure, PVP, which was present as a colloidal stabilizer, behaved as a soft template for chain growth [47], as described below. Notably, the shells appear to possess varying porosity. Consequently, it is plausible that $\mathrm{Co}^{2+}$ and $\mathrm{AuCl}_{4}{ }^{-}$ions continuously diffused through the shell during the galvanic replacement reaction and thereby gave rise to the formation of the hollow nanostructure.

As noted above, PVP is widely used as a colloidal stabilizer to inhibit the aggregation of metal nanoparticles such as gold [4952], silver [53,54], platinum [55-57], palladium [46,57-59], nickel $[60,61]$, and cobalt $[62,63]$. To verify this hypothesis in our system, we conducted the synthetic steps under similar conditions without adding any PVP. In the absence of PVP, the Co nanoparticles aggregated, leading to the formation of irregularly shaped particles as shown in Figure 6a. Additionally, we believe that PVP plays an important role in the formation of the chain structure; indeed, there have been some reports describing the formation of gold and nickel nanochains using PVP as a colloidal stabilizer $[60,64,65]$. Furthermore, in our experiments, we noted that the presence of the magnetic stirrer was also critical for the formation of the nanochains. Additional experiments to confirm the contribution of the magnetic stirrer were performed by swirling the solution by hand rather than using a magnetic stirrer. Figure 6b shows the TEM image of Au@Co nanoparticles prepared without using a magnetic stirrer. The TEM image shows discrete Au@Co nanospheres in contrast to the chain-linked structures obtained using a magnetic stirrer. This control experiment indicates a likely interaction between the magnetic nanoparticles and the external magnetic field produced by the magnetic stirrer during the galvanic replacement reaction. We hypothesize that both PVP and the use of a magnetic stirrer contribute to the assembly of these magnetic nanoparticles into nanochains.

\section{Optical properties of the Au@Co nanochains}

The optical properties of the Au@Co nanochains were characterized by UV-vis spectroscopy. Figure 7 shows the characteristic extinction spectrum of the Co nanoparticle solution before and after adding the $\mathrm{K}$-gold solution. No plasmon resonance peak was observed for the bare cobalt nanoparticles, and the extinction decreased with an increase in the wavelength as expected based on reports in the literature $[66,67]$. However, upon the addition of $\mathrm{K}$-gold solution, a plasmon resonance appeared as a strong peak at ca. $900 \mathrm{~nm}$; importantly, the nanochains possess no extinction bands characteristic of either bare gold nanoparticles or pure gold nanochains [68-70]. As mentioned above, $\mathrm{Au}$-coated hollow nanospheres using Co nanoparticles as a template exhibited a plasmonic resonance at $628 \mathrm{~nm}$ [27]. Consequently, our results represent a significant and potentially use-

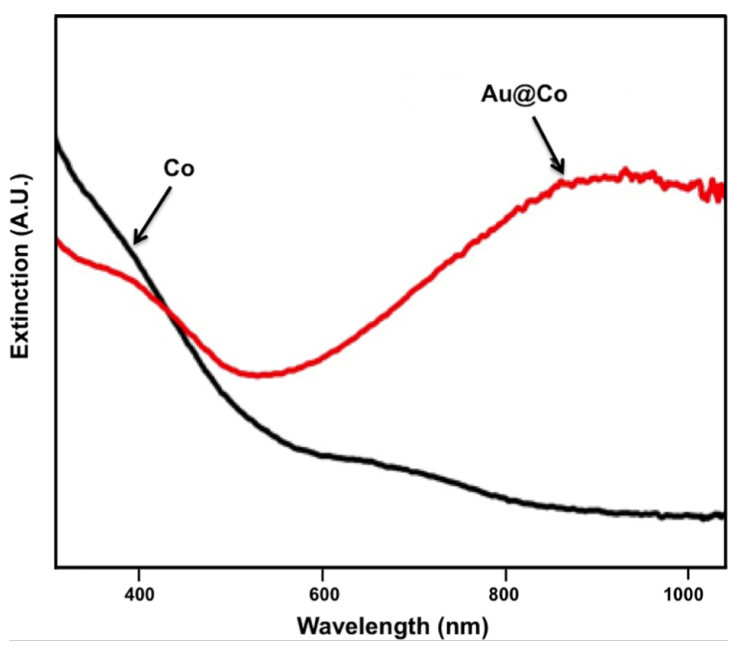

Figure 7: Extinction spectra of (a) Co nanoparticles and (b) Au@Co nanochains.
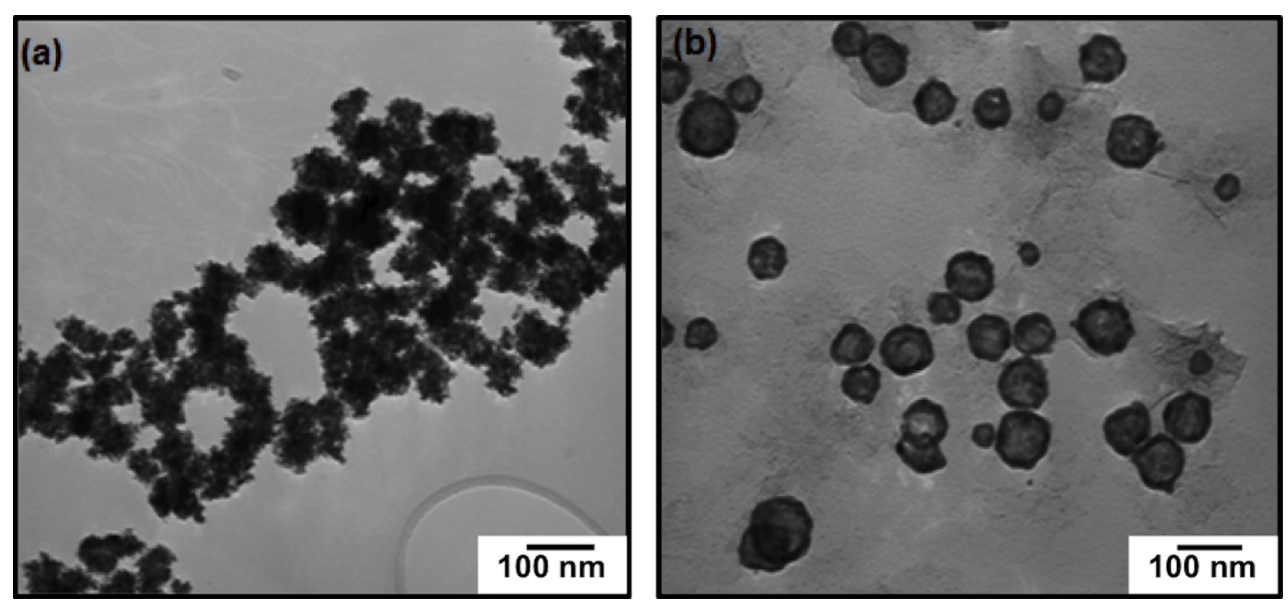

Figure 6: TEM images of Au@Co magnetic nanoparticles prepared (a) in the absence of PVP and (b) in the absence of a magnetic stirrer. 
ful outcome, as there have been, to our knowledge, no prior reports of NIR-responsive nanochain systems. There is a strong need for nanomaterials that are NIR-active [10-12,14], and our $\mathrm{Au} @ \mathrm{Co}$ nanochains help to fill in this need.

\section{Magnetic properties of the Au@Co nanochains}

In addition to their NIR extinction, our dual-functionality $\mathrm{Au} @$ Co nanochains possess magnetic properties, which were evaluated using a superconducting quantum interference device (SQUID) magnetometer. The temperature dependence of the magnetization for nanochains was measured in an applied field of $100 \mathrm{Oe}$ from 0 to $400 \mathrm{~K}$. Figure $8 \mathrm{a}$ shows the zero-field-cooling (ZFC) and field-cooled (FC) measurements of the Au@Co nanochains. The sharp maximum in the ZFC curve demonstrated the blocking behavior of the nanochains, with a blocking temperature of $150 \mathrm{~K}$ [71]. The field-dependent magnetization was measured at $5 \mathrm{~K}$ and is shown in Figure 8b; the magnetization is above $15 \mathrm{emu} / \mathrm{g}$ at a field of $60 \mathrm{kOe}$. No saturation was observed in the magnetization at $5 \mathrm{~K}$. The absence of a clear saturation of the Au@Co nanochains at low temperatures and high external fields, as seen in Figure 8b, suggests the presence of paramagnetism or a paramagnetic-like phase in the material. On the whole, the magnetic behavior observed in Figure $8 \mathrm{a}, \mathrm{b}$ is typical for superparamagnetic materials and suggests that the $\mathrm{Au} @$ Co nanochains are superparamagnetic.

\section{Conclusion}

This study reports the synthesis and characterization of goldcoated superparamagnetic Co nanochains with NIR extinction (ca. $900 \mathrm{~nm}$ ). We demonstrated the synthesis of magnetoopticalAu@Co nanochains using a modified wet-chemical method based on a galvanic replacement reaction and highlighted the significant roles of PVP and magnetic stirring in the formation of the chain structure. The Au@Co nanochains possess a strong optical extinction in the NIR spectral region, making them useful for several applications such as photo-thermally modulated drug delivery, photonic devices, and photothermal cancer therapy. In addition, these nanochains are superparamagnetic at room temperature, which offers the capacity for manipulation under an external magnetic field. Because of their dual magneto-optical properties, ourAu@Co nanochains can be used for both delivery and treatment, making them a unique new choice for biomedical applications in which such dual functional properties can be utilized.

\section{Experimental \\ Materials}

Cobalt chloride (Baker), polyvinylpyrrolidone $\left(M_{\mathrm{W}} \approx 50000\right.$, Aldrich), sodium borohydride (Aldrich), potassium carbonate (Aldrich), hydrogen tetrachloroaurate(III) hydrate (Strem), nitric acid (EM Science) and hydrochloric acid (EM Science) were purchased from the indicated suppliers and used without purification. Water was purified to a resistivity of $18 \mathrm{M} \Omega \cdot \mathrm{cm}$ (Academic Milli-Q Water System; Millipore Corporation). All glassware and equipment was cleaned using an aqua regia solution $\left(\mathrm{HCl} / \mathrm{HNO}_{3} 3: 1\right)$, thoroughly rinsed with Milli-Q water, and then dried prior to use. A basic solution of potassium and gold ( $\mathrm{K}$-gold solution) was prepared under vigorous stirring by adding $2 \mathrm{~mL}$ of $1 \% \mathrm{HAuCl}_{4} \cdot \mathrm{H}_{2} \mathrm{O}$ solution to $100 \mathrm{~mL}$ of Milli-Q water containing $0.025 \mathrm{~g}$ of potassium carbonate $\left(\mathrm{K}_{2} \mathrm{CO}_{3}\right)$. The mixture changed from yellow to colorless after the reaction had proceeded for an additional $30 \mathrm{~min}$. The $\mathrm{K}$-gold solution was stored under refrigeration in the dark for two days (with help of aluminum foil) until use.

\section{Synthesis of the Au@Co nanochains}

The synthesis of the nanochains proceeded using a wet-chemical method in which a reactor was charged with $8.5 \mathrm{mg}$ of $\mathrm{CoCl}_{2} \cdot 6 \mathrm{H}_{2} \mathrm{O}, 100 \mathrm{mg} \mathrm{PVP}$, and $50 \mathrm{~mL}$ of water under vigorous magnetic stirring (IKAMAG ${ }^{\circledR}$ C-Mag HS 7). The reaction was purged with Ar for $15 \mathrm{~min}$ and then a freshly prepared $5 \mathrm{~mL}$
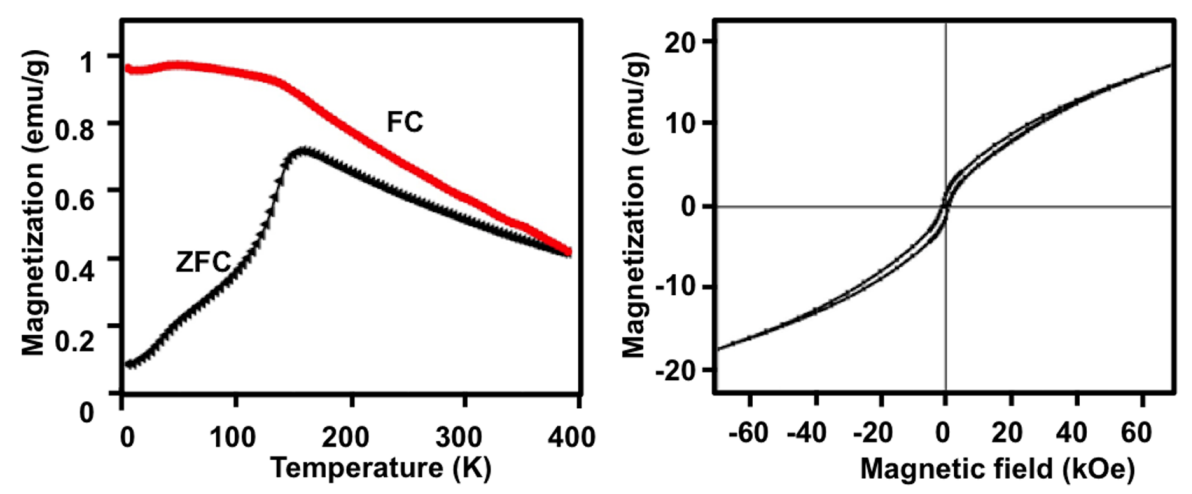

Figure 8: Magnetic properties of the Au@Co nanochains: (a) zero-field-cooling (ZFC), field-cooled (FC) at 100 Oe applied field, and (b) field-dependent magnetization $(M$ vs $H$ ) hysteresis loop. 
aqueous solution of $0.013 \mathrm{M} \mathrm{NaBH}_{4}$ was added dropwise. A light brown color was observed in the solution, indicating the formation of small cobalt nanoparticles [27]. An aliquot (5 mL) of K-gold solution was immediately added into the cobalt nanoparticle solution; subsequently, the color of the mixture changed from light brown to dark blue. The mixture was collected by centrifugation at $3000 \mathrm{rpm}$ for $30 \mathrm{~min}$ using an RC-3B refrigerated centrifuge (Sorvall Instruments) and redispersed in Milli-Q water prior to analysis.

\section{Characterization methods}

The size and morphology of the composite nanochains were characterized by field-emission scanning electron microscopy (FE-SEM) and TEM. FE-SEM measurements were carried out using a JEOL JSM 6330F instrument operating at an accelerating voltage of $15 \mathrm{kV}$. TEM measurements were carried out using a JEOL JEM-2000 FX electron microscope operating at an accelerating voltage of $200 \mathrm{kV}$. The samples were prepared by placing small drops of solution containing the dispersed nanochains on a silicon wafer (for FE-SEM) or on 300-mesh holey carbon-coated copper grids (for TEM) and allowing the solvent to evaporate.

Analysis by XRD was performed using a Siemens D-5000 with monochromatic $\mathrm{Cu} \mathrm{K} \alpha$ radiation $(\lambda=1.540562 \AA)$, and EDX measurements were collected using an Oxford EDX attached to the TEM microscope to confirm the structure. All extinction spectra were recorded at room temperature on a Cary 50 Scan UV-visible spectrometer over the wavelength range of 250-1100 nm. The magnetic properties of the nanochains were obtained using a SQUID magnetometer with fields up to $5 \mathrm{~T}$. The temperature-dependent magnetization curves varying between 5 and $400 \mathrm{~K}$ were measured in an applied magnetic field of $100 \mathrm{Oe}$.

\section{Acknowledgements}

We thank the Asian Office of Aerospace Research and Development (AFOSR/AOARD FA2386-16-1-4067), the Robert A. Welch Foundation (E-1320), and the Texas Center for Superconductivity for supporting this research.

\section{References}

1. Berry, C. C.; Curtis, A. S. G. J. Phys. D: Appl. Phys. 2003, 36, R198-R206. doi:10.1088/0022-3727/36/13/203

2. Grancharov, S. G.; Zeng, H.; Sun, S.; Wang, S. X.; O'Brien, S.; Murray, C. B.; Kirtley, J. R.; Held, G. A. J. Phys. Chem. B 2005, 109, 13030-13035. doi:10.1021/jp051098c

3. Tartaj, P.; del Puerto Morales, M.; Veintemillas-Verdaguer, S.; González-Carreño, T.; Serna, C. J. J. Phys. D: Appl. Phys. 2003, 36, R182-R197. doi:10.1088/0022-3727/36/13/202
4. Ji, X.; Shao, R.; Elliott, A. M.; Stafford, R. J.; Esparza-Coss, E.; Bankson, J. A.; Liang, G.; Luo, Z.-P.; Park, K.; Markert, J. T.; Li, C. J. Phys. Chem. C 2007, 111, 6245-6251. doi:10.1021/jp0702245

5. Jeong, U.; Teng, X.; Wang, Y.; Yang, H.; Xia, Y. Adv. Mater. 2007, 19, 33-60. doi:10.1002/adma.200600674

6. Alivisatos, A. P. Science 1996, 271, 933-937. doi:10.1126/science.271.5251.933

7. Schmid, G., Ed. Gluster and Colloids: From Theory to Applications; VCH: New York, NY, U.S.A., 1994. doi:10.1002/9783527616077

8. Kolhatkar, A. G.; Jamison, A. C.; Litvinov, D.; Willson, R. C.; Lee, T. R. Int. J. Mol. Sci. 2013, 14, 15977-16009. doi:10.3390/ijms140815977

9. Jain, P. K.; Xiao, Y.; Walsworth, R.; Cohen, A. E. Nano Lett. 2009, 9, 1644-1650. doi:10.1021/nl900007k

10. Linic, S.; Christopher, P.; Ingram, D. B. Nat. Mater. 2011, 10, 911-921. doi:10.1038/nmat3151

11. Clavero, C. Nat. Photonics 2014, 8, 95-103. doi:10.1038/nphoton.2013.238

12. Howes, P. D.; Chandrawati, R.; Stevens, M. M. Science 2014, 346, 1247390. doi:10.1126/science. 1247390

13. Shanmugam, V.; Selvakumar, S.; Yeh, C.-S. Chem. Soc. Rev. 2014, 43, 6254-6287. doi:10.1039/C4CS00011K

14. Dreaden, E. C.; Alkilany, A. M.; Huang, X.; Murphy, C. J.; El-Sayed, M. A. Chem. Soc. Rev. 2012, 41, 2740-2779. doi:10.1039/C1CS15237H

15. Yi, D. K.; Selvan, S. T.; Lee, S. S.; Papaefthymiou, G. C.; Kundaliya, D.; Ying, J. Y. J. Am. Chem. Soc. 2005, 127, 4990-4991. doi:10.1021/ja0428863

16. Santra, S.; Tapec, R.; Theodoropoulou, N.; Dobson, J.; Hebard, A.; Tan, W. Langmuir 2001, 17, 2900-2906. doi:10.1021/la0008636

17. Singh, R. K.; Kim, T.-H.; Patel, K. D.; Knowles, J. C.; Kim, H.-W. J. Biomed. Mater. Res., Part A 2012, 100, 1734-1742. doi:10.1002/jbm.a.34140

18. Malvindi, M. A.; De Matteis, V.; Galeone, A.; Brunetti, V.; Anyfantis, G. C.; Athanassiou, A.; Cingolani, R.; Pompa, P. P. PLoS One 2014, 9, e85835. doi:10.1371/journal.pone.0085835

19. Mayer, K. M.; Hafner, J. H. Chem. Rev. 2011, 111, 3828-3857. doi:10.1021/cr100313v

20. Haes, A. J.; Van Duyne, R. P. Expert Rev. Mol. Diagn. 2004, 4, 527-537. doi:10.1586/14737159.4.4.527

21. Levin, C. S.; Hofmann, C.; Ali, T. A.; Kelly, A. T.; Morosan, E.; Nordlander, P.; Whitmire, K. H.; Halas, N. J. ACS Nano 2009, 3, 1379-1388. doi:10.1021/nn900118a

22. Xu, Z.; Hou, Y.; Sun, S. J. Am. Chem. Soc. 2007, 129, 8698-8699. doi:10.1021/ja073057v

23. Alù, A.; Engheta, N. Opt. Express 2009, 17, 5723-5730. doi:10.1364/OE.17.005723

24. Weissleder, R. Nat. Biotechnol. 2001, 19, 316-317. doi:10.1038/86684

25. Bao, Y.; Calderon, H.; Krishnan, K. M. J. Phys. Chem. C 2007, 111, 1941-1944. doi:10.1021/jp066871y

26. Wetz, F.; Soulantica, K.; Falqui, A.; Respaud, M.; Snoeck, E.; Chaudret, B. Angew. Chem., Int. Ed. 2007, 46, 7079-7081. doi:10.1002/anie.200702017

27. Liang, H.-P.; Wan, L.-J.; Bai, C.-L.; Jiang, L. J. Phys. Chem. B 2005, 109, 7795-7800. doi:10.1021/jp045006f

28. Huang, W.-F.; Zhang, Q.; Zhang, D.-F.; Zhou, J.; Si, C.; Guo, L.; Chu, W.-S.; Wu, Z.-Y. J. Phys. Chem. C 2013, 117, 6872-6879. doi:10.1021/jp4009674

29. Duan, S.; Wang, R. Prog. Nat. Sci.: Mater. Int. 2013, 23, 113-126. doi:10.1016/j.pnsc.2013.02.001 
30. Lu, Y.; Zhao, Y.; Yu, L.; Dong, L.; Shi, C.; Hu, M.-J.; Xu, Y.-J.; Wen, L.-P.; Yu, S.-H. Adv. Mater. 2010, 22, 1407-1411. doi:10.1002/adma.200903298

31.Su, K.-H.; Wei, Q.-H.; Zhang, X.; Mock, J. J.; Smith, D. R.; Schultz, S. Nano Lett. 2003, 3, 1087-1090. doi:10.1021/nl034197f

32. Zhu, S.-Q.; Zhang, T.; Guo, X.-L.; Shan, F.; Zhang, X.-Y. J. Nanomater. 2014, 2014, 736165. doi:10.1155/2014/736165

33. Larson, T. A.; Bankson, J.; Aaron, J.; Sokolov, K. Nanotechnology 2007, 18, 325101. doi:10.1088/0957-4484/18/32/325101

34. Lim, J.; Tilton, R. D.; Eggeman, A.; Majetich, S. A. J. Magn. Magn. Mater. 2007, 311, 78-83. doi:10.1016/j.jmmm.2006.10.1169

35. Peng, S.; Lei, C.; Ren, Y.; Cook, R. E.; Sun, Y. Angew. Chem., Int. Ed. 2011, 50, 3158-3163. doi:10.1002/anie.201007794

36. Song, Y.; Modrow, H.; Henry, L. L.; Saw, C. K.; Doomes, E. E.; Palshin, V.; Hormes, J.; Kumar, C. S. S. R. Chem. Mater. 2006, 18, 2817-2827. doi:10.1021/cm052811d

37. Barreca, D.; Massignan, C.; Daolio, S.; Fabrizio, M.; Piccirillo, C.; Armelao, L.; Tondello, E. Chem. Mater. 2001, 13, 588-593. doi:10.1021/cm001041x

38. Vickerman, J. C. Surface Analysis - The Principle Techniques; Wiley: Chichester, United Kingdom, 1997.

39. Sun, Y.; Xia, Y. J. Am. Chem. Soc. 2004, 126, 3892-3901. doi:10.1021/ja039734c

40. Sun, Y.; Xia, Y. Nano Lett. 2003, 3, 1569-1572. doi:10.1021/nI034765r

41. Jin, Y.; Dong, S. J. Phys. Chem. B 2003, 107, 12902-12905. doi:10.1021/jp035400y

42. Srnová-Šloufová, I.; Lednický, F.; Gemperle, A.; Gemperlová, J. Langmuir 2000, 16, 9928-9935. doi:10.1021/la0009588

43. Sun, Y.; Mayers, B. T.; Xia, Y. Nano Lett. 2002, 2, 481-485. doi:10.1021/n1025531v

44. Gao, J.; Ren, X.; Chen, D.; Tang, F.; Ren, J. Scr. Mater. 2007, 57, 687-690. doi:10.1016/j.scriptamat.2007.06.049

45. Chen, J.; Wiley, B.; McLellan, J.; Xiong, Y.; Li, Z.-Y.; Xia, Y. Nano Lett. 2005, 5, 2058-2062. doi:10.1021/n1051652u

46. Xiong, Y.; Wiley, B.; Chen, J.; Li, Z.-Y.; Yin, Y.; Xia, Y. Angew. Chem., Int. Ed. 2005, 44, 7913-7917. doi:10.1002/anie.200502722

47. Guo, L.; Liang, F.; Wen, X.; Yang, S.; He, L.; Zheng, W.; Chen, C.; Zhong, Q. Adv. Funct. Mater. 2007, 17, 425-430. doi:10.1002/adfm.200600415

48. Vasquez, Y.; Sra, A. K.; Schaak, R. E. J. Am. Chem. Soc. 2005, 127, 12504-12505. doi:10.1021/ja054442s

49. Cheng, H.-W.; Huan, S.-Y.; Wu, H.-L.; Shen, G.-L.; Yu, R.-Q. Anal. Chem. 2009, 81, 9902-9912. doi:10.1021/ac9014275

50. Nakazato, Y.; Taniguchi, K.; Ono, S.; Eitoku, T.; Katayama, K. Phys. Chem. Chem. Phys. 2009, 11, 10064-10072. doi:10.1039/b915353e

51. Mishra, A.; Tripathy, P.; Ram, S.; Fecht, H.-J. J. Nanosci. Nanotechnol. 2009, 9, 4342-4347. doi:10.1166/jnn.2009.M57

52. Tan, Y.; Dai, X.; Li, Y.; Zhu, D. J. Mater. Chem. 2003, 13, 1069-1075. doi:10.1039/b211386d

53. Pietrobon, B.; Kitaev, V. Chem. Mater. 2008, 20, 5186-5190. doi:10.1021/cm800926u

54. Tsuji, T.; Thang, D.-H.; Okazaki, Y.; Nakanishi, M.; Tsuboi, Y.; Tsuji, M. Appl. Surf. Sci. 2008, 254, 5224-5230. doi:10.1016/j.apsusc.2008.02.048

55. Bonet, F.; Delmas, V.; Grugeon, S.; Herrera Urbina, R.; Silvert, P.-Y.; Tekaia-Elhsissen, K. Nanostruct. Mater. 1999, 11, 1277-1284. doi:10.1016/S0965-9773(99)00419-5
56. Park, J. Y.; Aliaga, C.; Renzas, J. R.; Lee, H.; Somorjai, G. A. Catal. Lett. 2009, 129, 1-6. doi:10.1007/s10562-009-9871-8

57. Teranishi, T.; Hori, H.; Miyake, M. J. Phys. Chem. B 1997, 101, 5774-5776. doi:10.1021/jp970599y

58. Ayyappan, S.; Srinivasa Gopalan, R.; Subbanna, G. N.; Rao, C. N. R. J. Mater. Res. 1997, 12, 398-401. doi:10.1557/JMR.1997.0057

59. Teranishi, T.; Miyake, M. Chem. Mater. 1998, 10, 594-600. doi:10.1021/cm9705808

60. Liu, X.; Guo, M.; Zhang, M.; Wang, X.; Guo, X.; Chou, K. Rare Met 2008, 27, 642-647.

61. Couto, G. G.; Klein, J. J.; Schreiner, W. H.; Mosca, D. H.; de Oliveira, A. J. A.; Zarbin, A. J. G. J. Colloid Interface Sci. 2007, 311, 461-468. doi:10.1016/j.jcis.2007.03.045

62. Kato, Y.; Sugimoto, S.; Shinohara, K.-i.; Tezuka, N.; Kagotani, T.; Inomata, K. Mater. Trans. 2002, 43, 406-409. doi:10.2320/matertrans.43.406

63. Platonova, O. A.; Bronstein, L. M.; Solodovnikov, S. P.; Yanovskaya, I. M.; Obolonkova, E. S.; Valetsky, P. M.; Wenz, E.; Antonietti, M. Colloid Polym. Sci. 1997, 275, 426-431. doi: $10.1007 / \mathrm{s} 003960050100$

64. Zhou, W.; He, L.; Cheng, R.; Guo, L.; Chen, C.; Wang, J. J. Phys. Chem. C 2009, 113, 17355-17358. doi:10.1021/jp906234n

65. Zhang, D.-F.; Niu, L.-Y.; Jiang, L.; Yin, P.-G.; Sun, L.-D.; Zhang, H.; Zhang, R.; Guo, L.; Yan, C.-H. J. Phys. Chem. C 2008, 112, 16011-16016. doi:10.1021/jp803102h

66. Petit, C.; Pileni, M. P. J. Magn. Magn. Mater. 1997, 166, 82-90. doi:10.1016/S0304-8853(96)00621-X

67. Podlaha, E. J.; Henry, L.; Guo, Z. ECS Trans. 2006, 1, 63-69. doi:10.1149/1.2214586

68. El-Sayed, I. H.; Huang, X.; El-Sayed, M. A. Nano Lett. 2005, 5, 829-834. doi:10.1021/nl050074e

69. Hussain, I.; Brust, M.; Barauskas, J.; Cooper, A. I. Langmuir 2009, 25, 1934-1939. doi:10.1021/la804207y

70. Polavarapu, L.; Xu, Q.-H. Langmuir 2008, 24, 10608-10611. doi:10.1021/la802319c

71. Chen, D.; Liu, S.; Li, J.; Zhao, N.; Shi, C.; Du, X.; Sheng, J. J. Alloys Compd. 2009, 475, 494-500. doi:10.1016/j.jallcom.2008.07.115

\section{License and Terms}

This is an Open Access article under the terms of the Creative Commons Attribution License (http://creativecommons.org/licenses/by/4.0), which permits unrestricted use, distribution, and reproduction in any medium, provided the original work is properly cited.

The license is subject to the Beilstein Journal of Nanotechnology terms and conditions: (http://www.beilstein-journals.org/bjnano)

The definitive version of this article is the electronic one which can be found at:

doi:10.3762/bjnano. 8.168 